\title{
The null energy condition in dynamic wormholes
}

\author{
David Hochberg ${ }^{+}$and Matt Visser ${ }^{++}$ \\ ${ }^{+}$Laboratorio de Astrofísica Espacial y Fúsica Fundamental, Apartado 50727, 28080 Madrid, Spain \\ ${ }^{++}$Physics Department, Washington University, Saint Louis, Missouri 63130-4899, USA
}

(17 February 1998; Revised 30 April 1998)

\begin{abstract}
We extend previous proofs that violations of the null energy condition (NEC) are a generic and universal feature of traversable wormholes to completely non-symmetric time-dependent wormholes. We show that the analysis can be phrased purely in terms of local geometry at and near the wormhole throat, and do not have to make any technical assumptions about asymptotic flatness or other global properties. A key aspect of the analysis is the demonstration that time-dependent wormholes have two throats, one for each direction through the wormhole, and that the two throats coalesce only for the case of a static wormhole.
\end{abstract}

Introduction: The fact that traversable wormholes are accompanied by unavoidable violations of the null energy condition (NEC) is perhaps one of the most central features of wormhole physics [1]3]. The original proof of the necessity for NEC violations at or near the throat of a traversable wormhole was limited to the static spherically symmetric Morris-Thorne wormhole [1], though it was rapidly realized that NEC violations typically occurred in at least some explicit examples of static non-symmetric [4] and spherically-symmetric timedependent [5] wormholes. A considerably more general proof of the necessity of NEC violations was provided by the topological censorship theorem of Friedman, Schleich, and Witt [6] though this theorem requires many technical assumptions concerning asymptotic flatness and causality conditions that limit its applicability.

We have recently adopted a different strategy and sought to develop new general theorems concerning energy condition violations at and near the throat of traversable wormholes by focusing attention only on the local behaviour of the geometry at and near the throat, and discarding all assumptions about symmetry, asymptotic behaviour, and causal properties. (This strategy was inspired by the fact that there are many classes of objects that we would meaningfully wish to call wormholes that possess either trivial topology [3] or do not necessarily possess asymptotically flat regions [7].) Such a strategy first requires that we develop robust and general definitions of what it means to be a wormhole throat.

Recently, we have succeeded in developing such definitions and theorems, first for the static but completely non-symmetric case [8,9], and secondly (as reported in this Letter) for the completely general time-dependent non-symmetric wormhole. Our central results are these:

(1) A time-dependent traversable wormhole will have two throats, one throat being associated with each direction of travel through the wormhole. These two throats coalesce into one in the case of a static traversable worm- hole but it is important to keep them distinguished in the time-dependent case.

(2) For each one of these throats the NEC is either violated or on the verge of being violated on the throat itself. (This is easy to prove.)

(3) For each one of these throats there will be an open region (topologically open in one of the null hypersurfaces passing through the throat) whose closure intersects the throat such that the NEC is violated throughout the open region. (Proving this requires a little more analysis.)

(4) For each one of these throats there will be an open interval such that the transverse averaged NEC (transverse averaged over the throat after it is pushed out in the appropriate null direction) is violated throughout the open interval. (Proving this requires a little more analysis.)

The proofs are sketched below, and are based on an extension of an idea due to Page [10] whereby wormhole throats are viewed as anti-trapped surfaces in spacetime. Further technical details may be found in 11, which also extends the present analysis to spacetimes including nonzero torsion.

We note that there are a number of published papers which claim to construct wormholes without NEC violations. These claims are most often based on semantic confusion, though sometimes actual computational errors have crept in, and we shall discuss the situation more fully in our conclusions.

Basic Definitions: Null geodesic congruences. Consider a compact two-dimensional spacelike hypersurface (denoted $\Sigma$ ) embedded in $(3+1)$-dimensional spacetime. (More precisely, a compact two dimensional orientable manifold that is embedded in spacetime in a two-sided and spacelike manner.) At each point on the hypersurface there are two null vectors orthogonal to the hypersurface, and these two null vectors can be extended to two null geodesic congruences (vector fields $l_{ \pm}$) that are well defined on an open neighborhood of the hypersurface 12,13 . Coordinates on the hypersurface will be denoted $x$, while the null geodesic congruences can be used to set up null coordinates $u_{ \pm}$on the orthogonal hyperplanes. The \pm labels are often denoted "ingoing" and "outgoing" though these labels are prejudicial and in the case of non-trivial topology are actually meaningless, the key point is that there are two possible null directions to travel along.

For each one of these null geodesic congruences one can define expansion, shear, and vorticity, in a manner completely analogous to standard textbook discus- 
sions 12,13. In particular, we have a pair of Raychaudhuri equations, one for each null geodesic congruence, so that:

$$
\frac{d \theta_{ \pm}}{d u_{ \pm}}=-\frac{1}{2} \theta_{ \pm}^{2}-\sigma^{ \pm a b} \sigma_{ \pm a b}+\omega^{ \pm a b} \omega_{ \pm a b}-R_{c}^{d} l_{ \pm}^{c} l_{ \pm d} .
$$

We now define a wormhole throat by demanding that at a throat the cross-sectional area of a bundle of light rays passing orthogonally through the putative throat should be at a strict local non-zero minimum. We implement this notion by first defining the hypersurfaces $\Sigma\left(u_{ \pm}(x)\right)$ to be the spacelike hypersurfaces formed by taking $\Sigma \equiv$ $\Sigma(0)$ and pushing it out an affine distance $u_{ \pm}(x)$ along the null congruence $l_{ \pm}$. Define the area in terms of the two-metric $\gamma$ induced on the hypersurface $\Sigma$ by

$$
A\left[\Sigma\left(u_{ \pm}(x)\right)\right]=\int_{\Sigma\left(u_{ \pm}(x)\right)} \sqrt{\gamma\left(x, u_{ \pm}(x), u_{\mp}=0\right)} d^{2} x .
$$

If $\Sigma$ is to be regarded as a throat, we must demand first that $A[\Sigma]>0$ and second that there exists some open neighborhood $\mathcal{U}$ surrounding the zero function $u_{ \pm}(x)=0$ such that on this open set

$$
A\left[\Sigma\left(u_{ \pm}\right)\right] \geq A[\Sigma] .
$$

Dropping the \pm to simplify notation this implies first that $\delta A /\left.\delta u\right|_{(u=0)}=0$, and secondly that $\delta^{2} A /\left.\delta u^{2}\right|_{(u=0)} \geq 0$. But the null variational derivatives of the cross-sectional area can be related to the extrinsic curvature of the hypersurface $\Sigma$, where $\Sigma$ is to be considered as a submanifold of the null hypersurface generated by the null congruence passing through $\Sigma$. That is, $\Sigma$ is a throat with respect to the null congruence $l_{ \pm}$provided first that the expansion of the null congruence vanishes everywhere on the throat (the extrinsic curvature is zero)

$$
\theta_{ \pm}=0
$$

and secondly that everywhere on the throat the expansion of the null congruence satisfies the (simple) "flare out" condition

$$
\frac{d \theta_{ \pm}}{d u_{ \pm}} \geq 0
$$

As it stands, this preliminary and simple definition of flare-out just marginally fails to distinguish a throat from a trapped surface, and so the equations (7) and (8) apply to throats as well to apparent horizons (and apply in fact to arbitrary cross sections of the apparent horizon of a dynamic black hole). We refine and strengthen our flareout definition below so as to exclude apparent horizons. This preliminary definition is already enough to prove the first two of our key results.

Theorems: First we note that a hypersurface that is extremal with respect to one of the null congruences (say $l_{+}$) will in general not be extremal with respect to the other. This is particularly clear in the case of a spherically symmetric but time-dependent wormhole where the two radial null geodesic congruences are clearly of paramount importance. Looking for the zero of $\theta_{+}$defines the wormhole throat for light travelling in the $l_{+}$ direction, looking for the zero of $\theta_{-}$defines the wormhole throat for light moving in the $l_{-}$direction. In the special case where the wormhole is static (not necessarily spherically symmetric) the two null vectors can be decomposed as $l_{ \pm}=(V \pm n) / 2$, where $V$ is a unit vector parallel to the time-translation Killing vector and $n$ is a unit spacelike vector normal to $\Sigma$ and orthogonal to $V$. With this notation the expansion can be computed in terms of the Lie derivative of the induced two-tensor on $\Sigma$ and is seen to be

$$
\begin{aligned}
\theta_{ \pm} & =\frac{1}{2} \operatorname{Tr}\left[\mathcal{L}_{l_{ \pm}} \gamma\right] \\
& =\frac{1}{4}\left(\operatorname{Tr}\left[\mathcal{L}_{V} \gamma\right] \pm \operatorname{Tr}\left[\mathcal{L}_{n} \gamma\right]\right) \\
& = \pm \frac{1}{4} \operatorname{Tr}\left[\mathcal{L}_{n} \gamma\right]
\end{aligned}
$$

This is completely in agreement with our previous analysis for the static case 8,9] and, since the vanishing of $\theta_{+}$now automatically implies the vanishing of $\theta_{-}$and vice versa, shows that in the static case the two throats coalesce into one.

Note that the two throats defined above share many of the properties more typically attributed to apparent horizons $[12,13]$. The throats can move around as a function of time in a rather complicated fashion and the three-surface swept out by the throats as a function of time need not necessarily be smooth, nor need this threesurface necessarily have a spacelike normal, though typically it will. Moreover, the relative motion of the two throats can be such as to render the wormhole either oneway traversable or two-way traversable (meaning from one throat to the other), depending on the degree of causal connectedness of the two three-surfaces $W\left(\Sigma_{ \pm}\right)$ swept out by the two throats. That is, one may have $I^{+}\left(\Sigma_{+}\right) \bigcap W\left(\Sigma_{-}\right) \neq \phi$, and/or $I^{+}\left(\Sigma_{-}\right) \bigcap W\left(\Sigma_{+}\right) \neq \phi$ or perhaps neither condition holding, where $I^{+}$denotes the chronological future.

Second, by applying the Raychaudhuri equation, using the fact that the twist $\omega_{ \pm}$is automatically zero for any orthogonal geodesic congruence, that $\sigma^{2} \geq 0$, and noting that by definition $\theta_{ \pm}=0$ and $d \theta_{ \pm} / d u_{ \pm} \geq 0$ at the throat, we immediately derive

$$
R_{a b} l_{ \pm}^{a} l_{ \pm}^{b} \leq 0
$$

where we must use the same null vector as is used in defining the throat. Applying the Einstein equations now yields

$$
T_{a b} l_{ \pm}{ }^{a} l_{ \pm}{ }^{b} \leq 0
$$

so that the NEC is either violated or on the verge of being violated at the throat $\left(T_{a b} l_{ \pm}{ }^{a} l_{ \pm}{ }^{b}\right.$ could be zero). Of course we want to derive a stronger result that replaces 
the weak inequality above by a strict inequality. Proving this is a little tedious and requires just a bit of mathematical analysis.

We supplement the preliminary definition of a throat given above with the condition that there be at least some (infinitesimal) variations for which the area is strictly increasing. More precisely suppose that there exists at least one $u_{ \pm}(x)=\epsilon f(x)$ with $\epsilon \in(-\delta, 0) \cup(0,+\delta)$ for which the area function satisfies the strict inequality

$$
A\left[\Sigma\left(u_{ \pm}=\epsilon f\right)\right]>A[\Sigma] .
$$

(As discussed more fully in [8,9,11], this constraint disposes of certain degenerate cases and guarantees that $\Sigma$ is a true local minimum of the area: there is at least one direction in which the area actually increases.) Application of the fundamental theorem of calculus now implies that there is an open interval in $\epsilon$ (in general $\left(-\delta_{2}, 0\right) \cup\left(0,+\delta_{2}\right)$, different from that above) for which

$$
\frac{d^{2} A\left[\Sigma\left(u_{ \pm}=\epsilon f\right)\right]}{d \epsilon^{2}}>0
$$

with this now being a strict inequality. Apparent horizons and bifurcation two-spheres fail to satisfy these strict inequalities and so are not examples of throats. In terms of the expansion this implies the existence of an open interval in $\epsilon$ (in general $\left(-\delta_{3}, 0\right) \cup\left(0,+\delta_{3}\right)$, different from that above) for which

$$
\int_{\Sigma\left(u_{ \pm}=\epsilon f\right)} d^{2} x \sqrt{\gamma} f^{2}(x) \frac{d \theta_{ \pm}}{d u_{ \pm}}>0
$$

This constraint on the expansion further implies the existence of another open neighborhood, this time in the appropriate null hypersurface passing through $\Sigma$ such that

$$
\left.\frac{d \theta_{ \pm}}{d u_{ \pm}}\right|_{\left(x, u_{ \pm}, u_{\mp}=0\right)}>0
$$

This last open set may include the hypersurface $\Sigma(0)$ itself but does not necessarily have to do so, its closure must however certainly intersect $\Sigma(0)$. These messy technical details are required to justify replacing the weak inequality by a strict inequality and occur in some form or another in all technical discussions of NEC violations in traversable wormholes. It is because of these technical complications that the phrase "at or near the throat" is ubiquitous. (See in particular the discussions in [8,9],11].)

Of course, with these incantations out of the way, the proof of NEC violations follows along the lines indicated previously and we find that there is an open neighborhood near the throat over which

$$
T_{a b} l_{ \pm}^{a} l_{ \pm}^{b}<0 .
$$

This completes the proof of the third key result enunciated above, while the fourth result follows from appropriate modifications of the integrated constraint Eq. (11). See [11] 14] for details.
Discussion: We have thus shown that the violations of the NEC commonly ascribed to traversable wormholes are completely generic - NEC violations will occur at or near the throat of any spacetime configuration that deserves to be called a (traversable) wormhole, and this result is completely general and independent of issues of symmetry, asymptotic flatness, or time dependence. The present discussion is completely in agreement with the original Morris-Thorne analysis [1], the topological censorship theorem [6], and our own earlier analyses of the generic static wormhole [8,9].

On the other hand, the striking nature of these NEC violations has led to a minor industry of papers claiming to construct wormholes without violating the energy conditions. We can classify these attempts into three classes:

(1) playing semantic games, (2) genuine ambiguities, and (3) simple error.

One way of playing semantic games is to arbitrarily divide the total stress energy into an "exotic component" plus a "normal component". As seen above, the total stress energy must always violate the NEC, but it is sometimes possible to force all the NEC violations into the "exotic component" of the stress-energy and keep the "normal component" well-behaved. This strategy of semantic confusion underlies all the claims that BransDicke wormholes do not violate the NEC, since for suitable choice of the $\omega$ parameter the Brans-Dicke scalar can be coerced into providing the NEC violations. (Of course, the fact that for certain values of the $\omega$ parameter Brans-Dicke gravity supports traversable wormhole solutions is both interesting and non-trivial, it is only the claimed lack of NEC violations that we find problematical.) Some papers adopting this strategy are [15], and we have provided a fuller treatment of the situation in 9]. The same sort of comments should be borne in mind for wormholes based on other variants of Einstein gravity, whether they be higher-derivative gravity [16], dilaton gravity, scalar-tensor gravity, or whatever. (We mention that adding torsion actually makes the problem worse not better, see [11].)

Another technique commonly used is to rely on time dependence to temporarily and locally suspend the violations of the NEC. Time-dependent wormholes in an inflationary context were first studied by Roman 17] (see also 18 ), who correctly recognized the existence of NEC violations. Subsequent papers have sought to utilize time-dependence to evade the NEC violations [19,22], and some commentary regarding these attempts can be found in [9, 11]. The key here is to note that the suspension of the NEC is essentially an illusion in that if one ever succeeds in passing through the wormhole and reaching the other universe then one must have passed a throat as defined above and there must be NEC violations at or near this throat. The NEC violations can be moved around to some extent, but if the NEC violations are suspended, then the ingoing null congruence is still contracting. A subtlety that has helped cause confusion in the past is that (as we have seen in this Letter) 
time-dependent wormholes typically have two throats. If the wormhole is symmetric under interchange of the two universes it connects, then these two throats will also be symmetrically placed but they will not be at the center of symmetry of the wormhole. It is only for static wormholes that the two throats coalesce. (This fact makes it easy to get confused about what it means to "pass through" a time-dependent wormhole - it is not enough to merely pass the central symmetry point. In passing through an expanding wormhole, the throat [extremal area] is encountered before reaching the center, while for a contracting wormhole the throat is encountered after crossing the center.)

Another source of confusion associated with past analyses of time-dependent wormholes arises from the use of spatial embedding techniques for defining and locating throats. These techniques require selecting and lifting out a particular time-slice from the given spacetime believed to contain a wormhole and then embedding this instantaneous three-geometry in a flat Euclidean $\mathcal{R}^{n}$. For a static wormhole, any constant time-slice will suffice and flare-out in the spatial embedding direction orthogonal to the throat is sufficient to imply flare-out in spacetime. But for a dynamic wormhole, where the two-dimensional throats sweep out complicated extended three-dimensional objects in spacetime, flare-out in the embedding direction (typically spacelike) does not imply flare-out in the null directions orthogonal to the twodimensional throat(s). (Computation has also convinced us that direct search for some extremal three-surface with spacelike normal is useless for deriving NEC violation theorems - the technical deficiency of such an approach is that without a natural definition of the "time" direction it is impossible to define what one means by null vectors orthogonal to a timelike three-surface.) Simple spatial embedding constructions also completely miss detecting the presence of the two throats.

Finally, we mention that a distressing number of papers addressing this topic are marred by actual calculational error, and encourage interested readers to proceed with caution.

In summary, we feel that the original observation by Morris and Thorne that traversable wormholes are accompanied by NEC violations has now been successfully and completely generalized to arbitrary traversable wormholes. The subtleties involved in the generalization to the time-dependent case lie in the fact that there are in general two wormhole throats for time-dependent traversable wormholes, and that some careful technical steps must be taken in the analysis in order to get the desired strong inequality (a weak inequality is much easier to derive). For generic static wormholes, the GaussCodazzi decomposition of the curvature tensor enables us to make considerably more detailed statements as to the curvature of spacetime near the throat.

Acknowledgements: This work was supported by Spain's National Aerospace Technology Institute (INTA) (D.H.), and by the US Department of Energy (M.V.).
M.V. acknowledges interesting and constructive comments made by Sean Hayward.

[1] M.S. Morris and K.S. Thorne, Am. J. Phys. 56, 395 (1988).

[2] M.S. Morris, K.S. Thorne and U. Yurtsever, Phys. Rev. Lett. 61, 1446 (1988).

[3] M. Visser, Lorentzian Wormholes: From Einstein to Hawking (American Institute of Physics, New York, 1995).

[4] M. Visser, Phys. Rev. D 39, 3182 (1989).

[5] M. Visser, Nucl. Phys. B 328, 203 (1989).

[6] J.L Friedmann, K. Schleich and D.M. Witt, Phys. Rev. Lett. 71, 1486 (1993).

[7] D. Hochberg, A. Popov and S.V. Sushkov, Phys. Rev. Lett. 78, 2050 (1997).

[8] D. Hochberg and M. Visser, Phys. Rev. D. 56, 4745 (1997).

[9] M. Visser and D. Hochberg, "Generic Wormhole Throats", in The Internal Structure of Black Holes and Spacetime Singularities, ed. L.M. Burko and A. Ori, (Institute of Physics, Bristol, 1997).

[10] D. Page, cited as a note-added-in-proof in [1].

[11] D. Hochberg and M. Visser, Dynamic wormholes, antitrapped surfaces, and energy conditions, gr-qc/9802046.

[12] S.W. Hawking and G.F.R. Ellis, The Large Scale Structure of Space-Time (Cambridge University Press, Cambridge, England, 1973).

[13] R.M. Wald, General Relativity (University of Chicago Press, Chicago, 1984).

[14] The averaged flare-out condition places a constraint on the putative throat by asking that the extremal surface be outward-flaring over at least half its area and must be carefully formulated to remain invariant under arbitrary affine reparametrizations of the null geodesic congruence. See 11] for more details.

[15] P.S. Letelier and A. Wang, Phys. Rev. D48, 631 (1993); A. Agnese and M. LaCamera, Phys. Rev. D51, 2011 (1995); L.A. Anchordoqui, S.E. Perez Bergliaffa and D.F. Torres, Phys. Rev. D55, 5226 (1997); K.K. Nandi, B. Bhattacharjee, S.M.K. Alam, and J. Evans, Phys. Rev. D57, 823 (1998);

[16] B. Bahwal and S. Kar, Phys. Rev. D. 46, 2464 (1992).

[17] T. Roman, Phys. Rev. D. 47, 1370 (1993).

[18] D. Hochberg and T. Kephart, Phys. Rev. Lett. 70, 2665 (1993).

[19] S. Kar, Phys. Rev. D. 49, 862 (1994).

[20] S. Kar and D. Sahdev, Phys. Rev. D. 53, 722 (1996).

[21] A. Wang and P.S. Letelier, Prog. Theor. Phys. 94, 137 (1995).

[22] L.A. Anchordoqui, D.F. Torres, M.L. Trobo, and S.E. Perez Bergliaffa, Phys. Rev. D57, 829 (1998). 